\title{
Estrategia ambiental para prevenir la tala ilegal en el parque nacional Yasuní, Ecuador. 2020
}

\author{
Washington Francisco Suarez Saltos \\ ab_solucionesjuridicas@ hotmail.com \\ Escuela De Posgrado: Programa Académico \\ Doctorado En Gestión Pública Y Gobernabilidad \\ Universidad Cesar Vallejo \\ Piura - Perú
}

\section{RESUMEN}

El presente trabajo de investigación abordó la temática referida a la tala ilegal que cada día crece y lleva consigo una serie de afectaciones al medioambiente, pero también a la población en su conjunto, este trabajo tuvo como objetivo Proponer una estrategia ambiental para prevenir la tala ilegal en el Parque Nacional Yasuní, Ecuador. La metodología tuvo un propósito como Aplicado, enfoque mixto y un alcance Descriptivo y Explicativo, donde el diseño fue no experimental de corte transversal, se obtuvo una muestra de 150 personas, profesionales vinculados al accionar legal y las políticas ambientales que laboran en el ministerio del ambiente y fiscalía. Los resultados mostraron que los procesos planteados en la planificación no están respondiendo a una estrategia ambiental viable, respecto a las estrategias ambientales estas no están promoviendo un constante monitoreo tecnológico ni tampoco un desarrollo ordenado de la población. Se concluye que una Estrategia Ambiental ayudaría significativamente a prevenir la Tala Ilegal en El Parque Nacional Yasuní, Ecuador, y que, de acuerdo con la opinión de especialistas sobre esta propuesta, arrojaron un Coeficiente de validez de contenido es 0.908 por lo que se recomienda la pertinencia de implementar y aplicar el programa.

Palabras Clave: estrategia ambiental; tala; ilegalidad; tala ilegal; deforestación. 


\title{
Environmental strategy to prevent illegal logging in Yasuní national park, Ecuador. 2020
}

\begin{abstract}
The present research work addressed the issue related to illegal logging that grows every day and carries with it a series of effects on the environment, but also on the population as a whole, this work aimed to propose an environmental strategy to prevent illegal logging in the Yasuní National Park, Ecuador. The methodology had a purpose such as Applied, mixed approach and a Descriptive and Explanatory scope, where the design was non-experimental, cross-sectional, a sample of 150 people was obtained, professionals linked to legal action and environmental policies that work in the ministry of environment and prosecution. The results showed that the processes proposed in the planning are not responding to a viable environmental strategy, with respect to environmental strategies they are not promoting constant technological monitoring or an orderly development of the population. It is concluded that an Environmental Strategy would significantly help prevent Illegal Logging in the Yasuní National Park, Ecuador, and that, according to the opinion of specialists on this proposal, they yielded a content validity coefficient of 0.908 , so the recommendation is relevance of implementing and applying the program.
\end{abstract}

KeyWords: environmental strategy; logging; illegality; illegal logging; deforestation.

Artículo recibido: 10 Agosto. 2021 Aceptado para publicación: 07. Setiembre. 2021 Correspondencia: ab_solucionesjuridicas@ hotmail.com Conflictos de Interés: Ninguna que declarar 


\section{INTRODUCCIÓN.}

Las cifras que se ha obtenido del último informe que fue emitido por Global Forest Watch, que es un entorno virtual de seguimiento de bosques en línea y a tiempo real cuyo propietario es el Instituto de Recursos Mundiales (World Resources Institute) cuya sede institucional se encuentra en Washington. Hace mención, el informe que el último se han perdido en trópicos varios millones de tierras o extensiones de bosques y que son analizados por la Universidad de Maryland, mostrando sus registros que 3,8 millones de hectáreas, es decir casi un tercio de la parte que se perdió correspondía a bosques primarios. Mostrando además que América Latina fue uno de lugares que más contribuyo en esta destrucción y ello fue en Brasil. De manera similar se emitió otro informe en el que se detalla que hay "masiva” pérdida de bosques en el país del altiplano boliviano. Y que a pesar que la cantidad fue menor que la que ocurrió de manera récord en los años 2016 y 2017, se comprueba que es tasa de pérdida, se posiciona como la tercera mayor en los últimos 20 años. (BBC News Mundo, 2020, párr. 1-7)

Según el reportaje realizado por de Costa (2020), nos comenta que el paisaje climático o bioma que alberga a la selva tropical más extensa y diversa del planeta tiene un área de más 6,8 millones de $\mathrm{km}^{2}$ y uno de sus múltiples propósitos consiste en apoyar a la región, y mantener equilibrado el clima, pero, los índices de deforestación en las nueve naciones amazónicas siguen en incrementándose. Bolivia, Brasil y Perú se posicionaron entre las cinco naciones que perdieron más bosques primarios en todo el planeta. (párr. 1-7)

Según Martín (2019), nos dice, que varios de los peores abusos cometidos en contra del medio ambiente y los derechos de la humanidad en el planeta son causados de una política extractiva de los recursos de la naturaleza y la corrupción en existe en el sistema económico y político a nivel mundial. Por lo que, si verdaderamente se quiere realizar planes para recuperar la ecología que vuelva a posicionar la salud, la seguridad, y el bienestar de las personas como centro del actuar, se debe tomar en consideración las principales causas de los ataques que se realizan en contra de las personas defensoras y por ende se debe seguir su ejemplo para proteger el medio ambiente y contener el cambio climático". La entidad mencionada destaca también la tendencia sin considerar proporción alguna de comunidades indígenas que han sido atacadas por proteger sus derechos y áreas territoriales, pese a que las investigaciones nos demuestran que éstas de 
manera conjunto con las comunidades, manipulan bosques que abarcan el carbono equivalente a, al menos, 33 veces las emisiones en el año, actualmente. (párr. 10-12)

De acuerdo a lo publicado por Luna (2018), nos menciona que la comunidad indígena Shawi, al enterarse que personas desconocidas estaban talando y llevándose ilegalmente los árboles más antiguos que forman parte de su territorio, en el mes agosto del 2018, en la parte nororiental de la Amazonía del Perú, citaron a una asamblea para analizar y definir qué acciones realizarían contra este grupo de taladores, además utilizaba la única carretera que unía a su comunidad con el distrito más próximo, Balsapuerto, utilizando esta vía carrozable para trasladar en camiones enormes los troncos de los árboles sacrificadas, y esto ocasionó a la vez, que por el gran peso que se trasladaba, se malograse la trocha. Los shawi instalaron un punto de control para no permitir el paso de los camiones, implementando una media que tiempo atrás el gobierno peruano la debió realizar para tener el control de las rutas usadas para el tráfico de madera, pero tal medida originó una represalia para sus dirigentes produciéndose una ola de amenazas violentas contra ellos, por haber tomado dicha decisión. (párr. 1-3)

Luna (2018), también informa que la ilegal poda realizada en la Amazonía incrementa de manera continuo de las áreas deforestadas. Pese a todo lo que se realiza por prevenir, nadie puede detener la destrucción de los bosques de las manos de mineros ilegales, ganaderos, agricultores individuales u otras formas de agricultura. En el territorio colombiano, el $70 \%$ de la perdida de los bosques se realiza en la Amazonía y aumentó $44 \%$, desde que salieron las FARC, siendo los más perjudicados, los bosques de la zona de Quibdó. En el caso de la selva de madre de Dios, en el Perú, la actividad de la minería ilegal está destruyendo el ecosistema en dicho territorio. En la Amazonía colombiana, la deforestación en distintos lugares tiene como protagonistas armados, que formaron parte de las FARC y de quienes crean vías para el narcotráfico para llegar hacia el Pacífico. En un artículo pequeño divulgado en la revista Science Advances casi a fines de febrero de 2018 y aludido por El Espectador, que es un viejo y gran científico comenta que los bosques de la Amazonía se acercaban a un punto de no retorno. Sus estimaciones cálculos sostienen que, en los 50 años últimos, toda la Amazonía, sin especificar fronteras de los 9 países que la conforman, han disminuido en un $17 \%$ de su vegetación. Advierte y afirma además que sí esta cifra llega al 20\%, la Amazonía, que es uno de los últimos pulmones verdes más significativos de la humanidad ya no será sostenible. (párr. 1-2) 
Lo antes descrito presenta un panorama muy desfavorable para la protección de árboles y de esta situación esperamos ver una luz de acción por parte del estado, frente a ello se ha formulado el problema de investigación consistente en ¿Cómo prevenir la tala ilegal en el Parque Nacional Yasuní, Ecuador. 2020?, esta problemática, se ha justificado considerando que las diferentes posiciones sobre el cuidado y protección de la tala ilegal, no solo es un problema de cortar madera y tratar de reemplazar con otra, es un tema que debemos conocer a mayor nivel de entendimiento integral sobre las repercusiones que esto genera, pues siendo conocedores de las causas y efectos que se producen al entorno de esta tala ilegal, cuanto afectamos al medioambiente y el grado de vulnerabilidad en que se encuentran las poblaciones que viven en el entorno. Esta justificación se realiza desde una perspectiva teórica, considerando que todo conocimiento existente sobre este tema ayudará a saber cuánto estamos aportando a medidas preventivas y correctivas sobre el accionar del hombre en desmedro de la producción maderera, el saber que los análisis sobre poder establecer recomendaciones que permitan incrementar el conocimiento sobre ello afianzarán los controles de protección. En la perspectiva práctica, se aplicarán las recomendaciones existentes, sobre el control de la tala ilegal y de cómo plantear respuestas inmediatas que articulen al estado y la población, así como al sector empresarial, puesto que en trabajos conjuntos ayuden a crear planes y directrices eficientes y eficaces. En cuanto a la perspectiva social, ésta se ha orientado a a lograr espacios territoriales donde primen los postulados de paz y tranquilidad, pues en espacios donde la corrupción campea en el día a día, la población vive en constante zozobra, es por ello que al plantear medidas de solución y el encaminamiento decidido a lograr consensos en pos del beneficio social, se logran espacios del buen vivir.

\section{Esto nos permite plantear como}

\section{Objetivo general}

- Proponer una estrategia ambiental para prevenir la tala ilegal en el Parque Nacional Yasuní, Ecuador. 2020. Y para lograr este cometido tenemos

\section{Objetivos específicos}

1. Diagnosticar el estado actual de la tala ilegal en el Parque Nacional Yasuní, Ecuador. 2020. 
2. Identificar los factores influyentes en la tala ilegal en el Parque Nacional Yasuní, Ecuador. 2020.

3. Diseñar la estrategia ambiental para prevenir la tala ilegal el Parque Nacional Yasuní, Ecuador. 2020, y

4. Estimar a través del juicio de expertos la pertinencia de implentar la estrategia ambiental sobre la tala ilegal en el Parque Nacional Yasuní, Ecuador. 2020.

\section{Este conjunto de situaciones nos permiten plantear la}

\section{Hipótesis}

- La implementación de una estrategia ambiental prevendría la tala ilegal en el Parque Nacional Yasuní, Ecuador. 2020.

\section{METODOLOGÍA.}

\subsection{Tipo y diseño de investigación.}

Según el propósito es aplicada, porque se expresa como una relación basada en aspectos y respaldo teórico existente, como las que se generaron por la manifestación de las ciencias puras y la forma en que se aplicó en los diversos momentos, permitiendo abarcar un espacio más que solo teorías abstractas. (Shuttleworth, 2008); según el enfoque es Mixta, porque realizan dos procesos complementarios que son observar y evaluar todos aquellos hechos fenomenológicos en la cual se plasman los fundamentos aclarados y al mismo tiempo determinados, hecho que nos permite demostrar ideas fundamentadas, base para plantear nuevos procesos de observación y evaluación. (Dzul, s/f); Según el alcance es Descriptiva, porque requiere enfatizar que característica puntual o variada se evidencia en la población y además delinea la forma natural el contexto demográfico sin cerrarse en razones, que motivan tal situación. (QuestionPro, 2021) y es Explicativa, porque lo descrito en base a conceptualizaciones siempre se dirigen a brindar respuestas sobre las causas de los fenómenos que se estudian en un determinado contexto. Asimismo, analiza que condición o condiciones se pueden estar presentando y trata de explicar por qué dos o más variables están relacionadas. (García, 2011). Así mismo en cuanto al Diseño de investigación fue no experimental, diseño seleccionado por contar con la característica de no manipular las variables en forma intencionada, el fenómeno es observado en su condición natural tal como ocurre, asimismo, la investigación fue de corte transversal, para la recolección los datos en un tiempo y momento únicos, y realizar un analices de su 
influencia y relación, abarcando grupos o subgrupos, (Hernández-Sampieri, Fernández y Baptista, 2010. p. 120).

\subsection{Variables y operacionalización.}

Variable Independiente, Estrategia Ambiental, la misma que está definida como el conjunto de acciones que tienen como objetivo esencial, mitigar los efectos medioambientales que se generan por la operación de las empresas industriales y de los derivados que producen. Considera la estrategia como la propuesta de un circuito analítico, en el que se formula e implantan diversas medidas protectivas ambientales y que tienen metas que se relacionan estrictamente con la rentabilidad. Bansal. (Bansal, 1997)

Variable Dependiente, Tala Ilegal de acuerdo con SNI ADEX (2017) nos dice que conceptualiza a la tala ilegal como a cualquier manera de afectación del bosque si contar con los permisos o afuera de algún sistema bajo regulación, que consienta la usabilidad responsable de las riquezas del bosque.

\subsection{Población, muestra, muestreo, unidad de análisis.}

Suárez (2011)La población es el conjunto de individualidades a las que se centra o dirige el estudio con la finalidad de comprender sus manifestaciones y aquello que se busca es poder razonar algo, delimitándola con criterios de inclusión y exclusión. En la investigación objeto de estudio se ha considerado como población a profesionales vinculados al accionar legal y a las políticas ambientales que laboran en el ministerio del ambiente y la fiscalía de prevención de delitos, establecidos en la ciudad de Guayaquil, quienes suman 150 en total.

La muestra La muestra está definida como un subgrupo de la población de estudio a la cual se pretende analizar y estimar valoraciones bajo los criterios de ser adecuada y representativa de esta. En el presente caso, al ser la población de estudio pequeña se ha considerado que la muestra a trabajar sea igual a la población, por lo tanto para esta investigación, $\mathrm{N}=\mathrm{n}=150$, lo que ha llevado a no realizar ningún tipo de muestreo.

\subsection{Técnicas e Instrumentos de recolección de datos.}

Toda investigación que se realiza respecto de una muestra de individuos, representativos de un colectivo grande, usa técnicas estandarizadas interrogativas, cuyo fin es obtener mediciones acerca de la diversidad de características bajo el contexto subjetivo y objetivo de la población. En el trabajo investigativo se utilizó la encuesta, donde se estableció 
preguntas de tipo estructuradas relacionadas a la variable de estudio, que permitió la obtención de información. En cuanto a los Instrumentos estos vienen hacer el medio que utilizará para guardar la información obtenida, el cuestionario es instrumento clásico a través del cual obtenemos y registramos datos. Su uso variable conlleva a usarlo como herramienta investigativa y como mecanismo para evaluar personas, procesos $\mathrm{y}$ programas formativos.

\subsection{Procedimientos}

Las fases que se realizaron para la recogida de los datos estuvieron dados por la aplicación del instrumento, es decir la encuesta que se le aplicó a los estudiantes y docentes, mediante aplicaciones Web como los formularios de google drive. Que permitió obtener información sobre las variables de estudios.

\subsection{Método de análisis de datos}

Una vez obtenidos los datos a través de las aplicaciones web respectivas, se procedió a la revisión minuciosa de las respuestas obtenidas, las que se tabularon mediante programas informáticos como SPSS, que es un software estadístico o a través del aplicativo Excel.

\subsection{Aspectos éticos}

Cada proceso investigativo de nivel académico, siempre deben estar sujetos a reglas que pueden determinar qué tan relevantes son los resultados obtenidos:

Respeto, aspecto ético importante dentro de una investigación en la cual se incluyen personas y por lo cual es imperante que se pueden generar situaciones legales o de relación con la moral, en tal sentido se consideran los derechos de quienes son considerados dentro de la investigación.

Transparencia, referido a la conducta humana, lo cual permite que todos comprendan de la forma más fácil el mensaje de lo que se quiere expresar, teniendo en consideración que ser transparente es ser claro, a tal manera de no dejar que se den malas interpretaciones.

Confiabilidad, referido a la capacidad de lograr realizar una función específica y que esta conlleve a generar confianza sobre los resultados hallados a través de procesos considerados adecuados y permitidos. 
Autonomía ejecutar un estudio investigativo, debe asegurarse de la independencia que el equipo investigador debe tener, pues los hallazgos a los que se arriben tendrán una connotación en la sociedad.

\section{RESULTADOS Y DISCUSIÓN}

\section{Tabla 1}

Los procesos planteados en la planificación están respondiendo a una estrategia ambiental viable

\begin{tabular}{ccc}
\hline Descripción & $\mathbf{f i}$ & $\mathbf{\%}$ \\
\hline TD & 52 & 34.67 \\
D & 59 & 39.33 \\
NO & 23 & 15.33 \\
A & 16 & 10.67 \\
\hline Total & $\mathbf{1 5 0}$ & $\mathbf{1 0 0 . 0 0}$ \\
\hline
\end{tabular}

\section{Fuente: El Autor}

En relación con los resultados de la Tabla 1, sobre si Los procesos planteados en la planificación están respondiendo a una estrategia ambiental viable, se manifiesta que un $34.67 \%$ manifiestan estar Totalmente en Desacuerdo se manifiesta que un $39.33 \%$ solo refieren estar en Desacuerdo con tal afirmación, asimismo un $15.33 \%$ prefirieron No Opinar, y finalmente un $10.67 \%$ ante dicha afirmación refieren estar de Acuerdo. Lo cual se corrobora con lo manifestado por Irving et al. (2021) nos muestra que el uso de una estrategia de muestreo ambiental durante la investigación de un brote transmitido por alimentos para respaldar la hipótesis de transmisión de un trabajador alimentario enfermo a los clientes de un restaurante. (p. 24). Cheng-Han y Snell (2021) exponen que las diferencias generadas entre las estrategias de CSD según el tema son muy necesarias porque ayudan a evidenciar la ubicación, el tiempo y el canal de información parecen reflejar cuatro factores: la presión para informar, la disponibilidad de buenas noticias, si una empresa estaba asumiendo la responsabilidad ética de abordar el tema y los posibles lectores. (p. 447). Chaudhry et al. (2020) muestran que es necesario sugerir a los gerentes de las empresas manufactureras o sectores similares introducir innovaciones en sus productos y procesos para desarrollar un mejor sistema EMA; deben proponer estrategias ambientales adecuadas para mejorar el desempeño de sus empresas. El estudio actual también tiende a ayudar a los formuladores de políticas a desarrollar políticas apropiadas 
para el sector manufacturero de Pakistán al darse cuenta de la importancia de la innovación ambiental, EMA y FES. (pp. 715-716)

\section{Tabla 2}

Las estrategias ambientales están promoviendo un constante monitoreo tecnológico

\begin{tabular}{ccc}
\hline Descripción & $\mathbf{f i}$ & \% \\
\hline TD & 40 & 26.67 \\
D & 47 & 31.33 \\
NO & 24 & 16.00 \\
A & 23 & 15.33 \\
TA & 16 & 10.67 \\
\hline Total & $\mathbf{1 5 0}$ & $\mathbf{1 0 0 . 0 0}$
\end{tabular}

\section{Fuente: El Autor}

En relación con los resultados de la Tabla 2, sobre si Las estrategias ambientales están promoviendo un constante monitoreo tecnológico, se manifiesta que un $26.67 \%$ manifiestan estar Totalmente en Desacuerdo y un $31.33 \%$ solo refieren estar en Desacuerdo con tal afirmación, asimismo un $16 \%$ prefirieron No Opinar, mientras que un $15.33 \%$ ante dicha afirmación refieren estar de Acuerdo y finalmente el $10.67 \%$ están Totalmente de Acuerdo con lo manifestado. Lo cual se corrobora con lo manifestado por Farghly et al. (2021) quienes concluyen que es de vital importancia realizar un constante monitoreo de las actividades y que el uso de las tecnologías ayuda a este proceso, pues la fuente de iluminación LED podría ser apropiada para la alteración de fuentes de luz incandescentes y fluorescentes en granjas de conejos para mejorar el rendimiento del crecimiento sin comprometer la carcasa y el bienestar del conejo. (p. 1). Fajardo (2017) refiere que esta investigación, tuvo como fin el que se visualice una de tantas posibilidades que se tiene para que las empresas manufactureras puedan prevenir el contaminar desde su origen, siendo necesario realizar monitoreos a través del uso de las tecnologías para prevenir la contaminación y el impacto ambiental de las emisiones que causan los procesos industriales que realizan. Paralelamente, determina lo que ganaría la organización con su implementación, ya que logra realizar un análisis que se inicia averiguando el origen de sus problemas ambientales, en todo ciclo de vida del producto, así como de los efectos dañinos de la disposición final de sus desechos. (pp. 47, 58)

\section{Tabla 3}


Las estrategias ambientales están generando un desarrollo ordenado en la población

\begin{tabular}{ccc}
\hline Descripción & fi & \% \\
\hline TD & 53 & 35.34 \\
D & 56 & 37.33 \\
NO & 26 & 17.33 \\
A & 15 & 10.00 \\
\hline Total & $\mathbf{1 5 0}$ & $\mathbf{1 0 0 . 0 0}$
\end{tabular}

\section{Fuente: El Autor}

En relación con los resultados de la Tabla 3, sobre si Las estrategias ambientales están generando un desarrollo ordenado en la población, se manifiesta que un $35.34 \%$ manifiestan estar Totalmente en Desacuerdo y un $37.33 \%$ solo refieren estar en Desacuerdo con tal afirmación, asimismo un $17.33 \%$ prefirieron No Opinar, mientras que un $10 \%$ ante dicha afirmación refieren estar de Acuerdo. Lo cual se corrobora con lo manifestado por Pino (2020) concluye que cogiendo como modelo a una entidad para averiguar el impacto económico que se genera en una organización el implantar las prácticas mencionadas, muestran como resultado una disminución significativa de emisiones de gases de invernadero, incremento de la eficiencia en el empleo de recursos, disminución de costos y la generación de un ahorro de \$ 84.073 al año con una inversión de inicio de $\$ 81.960$ que se recupera al segundo año. (p. x). Calvache Pejendino y Ceballos (2021) manifestaron el estudio enfocó dos estrategias: la primera que es la implementación de terrazas modulares y la segunda que es un sistema de movilidad sostenible, la propone el uso de la bicicleta como transporte, para mejorar la calidad ambiental en el entorno en el que se aplique. Concluyó el estudio, que el aplicar el índice de calidad ambiental urbana goza de tener una viabilidad moderada cuando se aplica al nivel estratégico de la comunidad. (p. 114)

\section{Tabla 4}

La tala ilegal viene creciendo como parte de una débil intervención policial

\begin{tabular}{ccc}
\hline Descripción & $\mathbf{f i}$ & \% \\
\hline D & 23 & 15.33 \\
NO & 30 & 20.00 \\
A & 55 & 36.67 \\
TA & 42 & 28.00 \\
\hline Total & $\mathbf{1 5 0}$ & $\mathbf{1 0 0 . 0 0}$ \\
\hline
\end{tabular}

\section{Fuente: El Autor}


En relación con los resultados de la Tabla 4, sobre si La tala ilegal viene creciendo como parte de una débil intervención policial, se manifiesta que un $15.33 \%$ solo refieren estar en Desacuerdo con tal afirmación, asimismo un $20 \%$ prefirieron No Opinar, mientras que un $36.67 \%$ ante dicha afirmación refieren estar de Acuerdo y finalmente el $28 \%$ están Totalmente de Acuerdo con lo manifestado. Estos resultados se pueden corroborar con lo vertido por Valdez y Cisneros (2020) quienes basados en los datos estadísticos recogidos en las entrevistas a actores clave y en las investigaciones de campo de la ámbito del estudio, se ha podido recalcar la importancia de la gobernanza ambiental en los ámbitos locales para lograr una mayor eficacia del resguardo forestal, así como realizar una reflexión sobre la existencia de una batalla de modelos asociados a la práctica de una relación de la naturaleza con el Buen Vivir, que de manera análoga se puede presentar como el extractivismo vs conservacionismo. (p. 148). Curz y Delgado (2018) Aprovechan el argumento de la denuncia pública para realizar un análisis superficial del actuar de la Procuraduría Federal de Protección al Ambiente y deja algunas conclusiones, que también podrían tomar como recomendaciones para estar seguros de que el Estado cumpla con su compromiso de poder garantizar un ambiente sano a sus pobladores (pp. 85-86). Rose (2017) El estudio de caso demuestra que trabas legales difíciles pueden obstaculizar la cooperación práctica entre países vecinos en la lucha contra los delitos ambientales transnacionales en el sector de la madera. Por lo tanto, es necesaria la armonización internacional de las leyes sobre delitos ambientales. (p. 128)

\section{CONCLUSIONES}

1. La presente investigación concluye que una propuesta sobre la implementación de una Estrategia Ambiental para prevenir la Tala Ilegal en El Parque Nacional Yasuní, Ecuador. 2020 es muy necesaria, la deforestación que se viene dando como consecuencia de la creciente tala ilegal está desencadenando en problemas no solo medioambientales, sino también económico y sociales, sobre los cuales el estado debe poner mayor interés.

2. Se identificaron situaciones muy relevantes que están incidiendo en el avance de esta problemática como lo es la tala ilegal y que están afectando el contexto global de la zona de intervención, mostrando la poca capacidad de intervención por parte del estado, la defraudación tributaria, la contaminación, la veda forestal ente otros. 
3. Se detectaron ciertos factores que tributan al incremento de esta actividad delictiva, y entre ellos encontramos las prácticas ilícitas que bajo la poca intervención del estado y de hacer imperar la ley y el orden solo se muestra indiferencia, la afectación al contexto económico como producto del contrabando, la evasión fiscal y la alteración medioambiental que destruye la biodiversidad de la zona, así como el incremento contaminante que genera esta actividad.

4. Se elaboró una estrategia que permite atender y enfrentar esta situación bajo un contexto planificado, con el fin de identificar los puntos neurálgicos a atender, el soporte tecnológico que apoyará oportunamente las intervenciones y acciones de control, situaciones que derivaran en el impacto social positivo que se pretende como meta y evidenciar la efectividad de dicho plan.

5. Los resultados estadísticos, a través del juicio de expertos, demuestran la necesidad de aplicar esta Estrategia ambiental, la cual ha sido revisada y evaluada por especialistas, conocedores de la gestión del estado y el análisis legal de intervención, arrojan un Coeficiente de validez de contenido es 0.908 con lo cual se determina la pertinencia de implementar y aplicar la estrategia.

\section{REFERENCIAS}

Amir, M. y Chaudhry, N. (2019). Linking Environmental Strategy to Firm Performance: A Sequential Mediation Model via Environmental Management Accounting and Top Management Commitment. Pakistan Journal of Commerce \& Social Sciences, 13(4), p. 849-867. https://www.econstor.eu/handle/10419/214255

Bárcena, A. (2020). Los Efectos Económicos y Sociales del COVID-19 en América Latina y el Caribe. CEPAL. Pág. 1 - 16. https://bit.ly/3rFG6vT

Belmira, M. (2018). Estrategias Metodológicas Para El Desarrollo Del Enfoque Ambiental. Estudio De Caso De Un Docente Del Área De Ciencia, Tecnología Y Ambiente De Una Institución Educativa Pública. Pontificia Universidad Católica Del Perú. p. 1-102. http://tesis.pucp.edu.pe/repositorio/handle/20.500.12404/13589

Bohan, C., Kaiyan, P., Parkinson, C., Bertozzi, A., Slough, T. y Johannes, U. (2021). Modeling illegal logging in Brazil. Research in the Mathematical Sciences, 8(2). http://dx.doi.org/10.1007/s40687-021-00263-6 
Bohan, K., Parkinson, A., Slough, T., y Urpelainen, J. (2021). Modeling illegal logging in Brazil Research in Mathematical Sciences, 8(2),29. p. 1-21. https://doi.org/10.1007/s40687-021-00263-6

Bonilla, M. Molina, J. Morales, F. (2006). Planificación: Tipos Y Herramientas. Universidad Fermin Toro. https://frankmorales.webcindario.com/trabajos/tiposplani.html

Burga, M. (2016). “Incremento de la Deforestación Y Sus Consecuencias en la Pérdida de Biomasa en los Bosques De La Provincia Alto Amazonas del Departamento De Loreto, 2000-2014”. Universidad Científica Del Perú. p. 1-75. http://repositorio.ucp.edu.pe/handle/UCP/107

Calvache, D., Pejendino J. y Ceballos, A. (2021). Estrategias Para El Mejoramiento De La Calidad Ambiental en La Comuna Tres, San Juan De Pasto. Revista de Investigación Agraria y Ambiental, 12(1), p. 113-132. https://doi.org/10.22490/21456453.3617

Carmona, F., Dal, G., Ramírez-Rodríguez, G., Pii, Y., Delgado-López, J., Guagliardi, A. y Masciocchi, N. (2021). Urea-functionalized amorphous calcium phosphate nanofertilizers: optimizing the synthetic strategy towards environmental sustainability and manufacturing costs. Scientific Reports, 11(1), p. 1-14. https://doi.org/10.1038/s41598-021-83048-9

Chaudhary, M. y Pathak, R. (2017). A dynamical approach to the legal and illegal logging of forestry population and conservation using taxation. Advances in Difference Equations, 2017(1), p. 1-23. https://doi.org/10.1186/s13662-017-1439-0

Chaudhry, N., Asad, H., Amir, M. y Hussian, R. (2020). Environmental Innovation and Financial Performance: Mediating Role of Environmental Management Accounting and Firm's Environmental Strategy. Pakistan Journal of Commerce \& Social Sciences, 14(3), p. 815-837. https://www.econstor.eu/handle/10419/224961

Cheng-Han, T. y Snell, R. (2021). Strategies for Social and Environmental Disclosure: The Case of Multinational Gambling Companies. Journal of Business Ethics, 168(3), p. 447-467. https://doi.org/10.1007/s10551-019-04190-z

Chiun-Jr, H., Fang-Hua, C., Yi-Shiang, H., Yu- Mei, H., Yu-Hsin, T., Chang-En, P., ChiHsiang, C., Yu-Shyang, C., Shau-Chian, L, You, Y., Shuo-Yu, H., Hsiang-Chih, H., Hsu, C., Meng-Yi, C., Ting-An, L., Hsin-Yi, S., Yu-Ching, T. y Chi-Tsong, C. 
(2020). Development and technical application of SSR-based individual identification system for Chamaecyparis taiwanensis against illegal logging convictions. Scientific Reports (Nature Publisher Group), 10(1), p. 1-15. http://dx.doi.org/10.1038/s41598-020-79061-Z

Comité Forestal SNI y Comité de Madera e Industria de la Madera ADEX (2017). ¿Qué se entiende por tala ilegal?. DIARIO GESTION. https://bit.ly/30DmfBL

Condé, T., Higuchi, N. y Lima, A. (2019). Illegal Selective Logging and Forest Fires in the Northern Brazilian Amazon. Forests, 10(1). http://dx.doi.org/10.3390/f10010061

Corporación Autónoma Regional de Cundinamarca (s/f). Características De La Gestión $\begin{array}{llll}\text { Ambiental. } & \text { Escuela }\end{array}$ http://elearning.sigci.car.gov.co/moodle/mod/book/view.php?id=6\&chapterid=67

Curz, E. y Delgado, C. (2018). La Sierra Tarahumara, Sin Justicia Ambiental. Boletín Mexicano de Derecho Comparado, nueva serie, año L, 151. p. 86 - 106. http://dx.doi.org/10.22201/iij.24484873e.2018.151.12290

Dat Tien, Wing-Keung, W., Moslehpour, M. y Quynh, H. (2019). Speculating Environmental Sustainability Strategy for Logistics Service Providers Based on Dhl Experiences. Journal of Management Information \& Decision Sciences, 22(4), p. 415-443. https://bit.ly/3vMo8d8

De Araujo, A. y Di Giulio, G. (2020). [Sustainable development: a narcissistic strategy to cope with the environmental crisis?] [Desenvolvimento sustentável: uma estratégia narcísica para enfrentar a crise ambiental?] [Desarrollo sostenible: ¿una estrategia narcisista para enfrentar la crisis ambiental?]. Ambiente e Sociedade, 23, p. 1-23. http://dx.doi.org/10.1590/1809-4422asoc20190050r3vu2020L6AO

Estévez, R. (2013). Medio Ambiente. Eco Inteligencia. https://www.ecointeligencia.com/2013/06/tipos-estrategia-ambiental-empresa/ ETHICSGLOBAL (2018). Las conductas ilícitas ¿qué son y de dónde vienen?. blog.ethicsglobal.com. https://bit.ly/3qKjdGm

Fajardo, H. (2017). La Producción más Limpia como Estrategia Ambiental en el Marco del Desarrollo Sostenible. Revista De Ingeniería, Matemáticas y Ciencias De La Información, 4(8). http://dx.doi.org/10.21017/rimci.2017.v4.n8.a32

Farghly, M., Mahrose, K., Peris, S., Abou-Kassem, D., Metwally, K., Abougabal, M. y Abd El-Aziz, A. (2021). Effects of lighting source as an environmental strategy for 
heat stress amelioration in growing Californian rabbits during summer season. Animal Biotechnology, p. 1-8. https://doi.org/10.1080/10495398.2021.1895186

Fernández, H. (s/f). ¿Qué es la tecnología y por qué es tan importante?. ECONOMIA TIC. https://economiatic.com/que-es-la-tecnologia/

FLRA (s/f). Practicas Ilicitas (ULP). Flra.gov. https://bit.ly/3qHRZ2X

Gestión en Recursos Naturales (2018). Impacto Ambiental. Grn.cl. https://bit.ly/3cvb3N2

Giva, A., Herman, N. y Mohd, O. (2020). Using Long-Range Wireless Sensor Network to Track the Illegal Cutting Log. Applied Sciences, 10(19), p. 6992. http://dx.doi.org/10.3390/app10196992

Gordon, S. (2016). The Foreign Corrupt Practices Act: Prosecute Corruption and End Transnational Illegal Logging. Boston College Environmental Affairs Law Review, 43(1), p. 111-144. https://bit.ly/3uMWBqF

Gutiérrez-Rua, J., Posada-García, M. Y González-Pérez, M. (2019). Prácticas de recursos humanos que impactan la estrategia de sostenibilidad ambiental. Innovar, 29(73), p. 11-24. http://dx.doi.org/10.15446/innovar.v29n73.78008

Hernández, D. (2018). Estrategia Curricular De Educación Ambiental Para Los Estudiantes De La Carrera Licenciatura en Educación Pedagogía-Psicología. Revista Luna Azul, 46, p. 369-386. https://doi.org/10.17151/luaz.2018.46.19

Interpol (2021). Productos ilegales - problemática. Interpol.int. https://bit.ly/3bHDCYc

Irving, D., Ripley, D., Shepherd, C., Waller, L., Pepper, J. y Dunn, J. (2021). Use of an Environmental Swabbing Strategy to Support a Suspected Norovirus Outbreak Investigation at a Retail Food Establishment. Journal of Environmental Health, 83(9), p. 24-26. https://searchworks.stanford.edu/articles/aph_149823524

Jackson, B., Decker, J., Brown, C. y Boyd, D. (2020). Understanding the co-occurrence of tree loss and modern slavery to improve efficacy of conservation actions and policies. Conservation Science and Practice, 2(5). http://dx.doi.org/10.1111/csp2.183 Jiunn-Cheng, L., Jun-yen, L. y Wan-Yu L. (2021). Risk analysis of regions with suspicious illegal logging and their trade flows. Sustainability, 13. p. $1-12$. https://doi.org/10.3390/su13063549 
Kieran, S., Hull, J. y Finger, A. (2021). Environmental DNA sampling provides new management strategies for vernal pool branchiopods in California. PLoS ONE, 16(4), p. 1-15. https://doi.org/10.1371/journal.pone.0243338

Kimutai, D. y Watanabe, T. (2016). Forest-Cover Change and Participatory Forest Management of the Lembus Forest, Kenya. Environments, 3(3). p. $1-18$. http://dx.doi.org/10.3390/environments3030020

Landero-Lozada, S., Toledo-Aceves, T., López-Barrera, F., Sosa, V. y Ramírez-Marcial, N. (2018). Early establishment of endangered and valuable tree species in cloud forest restoration plantings. Revista Mexicana de Biodiversidad 90. p. 1-12. https://doi.org/10.22201/ib.20078706e.2019.90.2550

Leal, C. (2019). Aguzar la mirada colectiva, el gran desafío de la historia ambiental latinoamericana. Historia y Sociedad (01218417), 36, p. 243-268. https://doi.org/10.15446/hys.n36.71970

Lende, S. (2018). Destrucción de Bosques Nativos y Deforestación Ilegal: El Caso Argentino (1998-2016). Papeles De Geografía, (64), p. 154-180. http://dx.doi.org/10.6018/geografia/2018/349551

Li, Y., Deng, Q., Zhou, C. y Feng, L. (2020). Environmental governance strategies in a two-echelon supply chain with tax and subsidy interactions. Annals of Operations Research, 290(1/2), p. 439-462. https://doi.org/10.1007/s10479-018-2975-z

Mojica-Macías, P., Ortíz-Moreno, L. y Gnecco-Lizcano, M. (2019). Estrategia De Gestión Ambiental Basada en Los Servicios Ecosistémicos Del Caño Siete Vueltas (Villavicencio, Colombia). Revista Luna Azul, 49, p. 38-63. https://doi.org/10.17151/luaz.2019.49.3

Mporas, I., Perikos, I., Kelefouras, V. y Paraskevas, M. (2020). Illegal Logging Detection Based on Acoustic Surveillance of Forest. Applied Sciences, 10(20), p. 7379. http://dx.doi.org/10.3390/app10207379

Naranjo, L. (2019). Conceptos relevantes en las estrategias ambientales en una empresa. USIL. https://bit.ly/3rL8Xis

Nazir, N. y Olabisi, L. (2017). Illegal Logging and Wood Consumption: Estimation and Projection of Illegal Wood Harvesting in Pakistan through System Dynamics. Pakistan Journal of Commerce \& Social Sciences, 11(2), p. 406-427. http://www.jespk.net/publications/375.pdf 
Niño, L. y Pedraza-Jiménez, Y. (2018). Potenciar la educación ambientala través del estudio de caso. Tecné, Episteme y Didaxis: ted, 45. p. 143 - 158. https://revistas.pedagogica.edu.co/index.php/TED/article/view/9839

Omal (s/f). Tipos de impactos. Aula critica. Empresas Transnacionales y derechos humanos, Materiales didácticos para repensar el modelo de desarrollo. Omal. https://omal.info/spip.php?article5645

Pabón, Y. y Miranda, J. (2010). CARACTERISTICAS DE LA PLANIFICACION. Caribbean International University. http://goyoplan701.blogspot.com/2010/06/caracteristicas-de-la-planificacion.html

Padilla, C. (2019). El impacto social es un cambio significativo y positivo que aborda un desafío acuciante. esimpact.org. https://bit.ly/3bJJX5F

Pérez, J. y Ana Gardey, A. (2018). Impacto Económico. Definición.de. https://bit.ly/3esH7Up

Pérez, J., \& Gardey, A. (2018). Definición de impacto social. definicion.de. https://definicion.de/impacto-social/

Pino, M. (2020). “Implementación de Prácticas Ambientales Sostenibles en Empresas Ecuatorianas y su Impacto Económico". Universidad Católica de Santiago de Guayaquil. p. 1 - 107. https://bit.ly/3vUmRAP

Puentestar, W. (2015). "La Problemática Ambiental y el Deterioro de los Recursos Naturales en el Ecuador. Una Perspectiva desde la Geografía". Pontificia Universidad Católica del Ecuador. p. 1 - 116. https://bit.ly/3cckDFd

Pymes El Financiero (2014). Seis prácticas ilegales y poco éticas que se cometen al hacer publicidad. elfinancierocr.com. https://bit.ly/2NfwDwh

Quishpe-López, J., Lliguicota-Guarquila, J., Sarduy-Pereira L. y Diéguez-Santana, K. (2020). La producción más limpia, como estrategia de valorización (ecoeficiencia) del centro de faenamiento, Puyo, Pastaza, Ecuador. 7.p. 59 - 71. https://revista.ucsact.edu.py/ojs/index.php/ucsa/article/view/54

Raffino, M. (2020). Conceptos de Deforestación. Conceptos.de. https://concepto.de/deforestacion/

Raffino, M. (2020). Crisis Economica. Conceptos.de. https://bit.ly/2Q0gb4a

Raffino, M. (2020). Tecnología. concepto.de.

https://concepto.de/tecnologia/\#ixzz6oqxDP79y 
curzte, A. (2020). Recepción de la ecoética en las Estrategias y Programas de Educación Ambiental: análisis comparativo entre España y Ecuador. Observatorio Medioambiental, 23, p. 165-186. http://dx.doi.org/10.5209/obmd.73175

Richardson, V. y Peres, C. (2016). Temporal Decay in Timber Species Composition and Value in Amazonian Logging Concessions. PLoS One, 11(7). http://dx.doi.org/10.1371/journal.pone.0159035

Rodriguez, L. (s/f). La estrategia medioambiental y la formación del profesional. Universidad de Holguín "Oscar Lucero Moya". Monografias.com. https://bit.ly/3eC8nQu

Rosales, M. (2020). La tala ilegal de árboles devora el Amazonas en Perú. Cambio 16. https://www.cambio16.com/tala-ilegal-de-arboles-amazonas-peruano/

Rose, G. (2017). Australian Law to Combat Illegal Logging in Indonesia: A Gossamer Chain for Transnational Enforcement of Environmental Law. Review of European Comparative \& International Environmental Law, 26(2), p. 128-138. https://doi.org/10.1111/reel.12206

Secretaría de Medio Ambiente y Recursos Naturales (2018). Impacto ambiental y tipos de impacto ambiental. Gobierno de Mexico. https://bit.ly/3rLv1tg

Socio Lab (2019). Características de un emprendimiento social. SocialabBlog. https://blog.socialab.com/que-es-emprendimiento-social/

Tereba, A., Woodward, S., Konecka, A., Borys, M. y Nowakowska, J. A. (2017). Analysis of DNA profiles of ash (0RW1S34RfeSDcfkexd09rT2Fraxinus excelsior1RW1S34RfeSDcfkexd09rT2 L.) to provide evidence of illegal logging. Wood Science and Technology, 51(6), p. 1377-1387. http://dx.doi.org/10.1007/s00226-017-0942-5

Tibanlombo, D. (2018). Tala y Comercio Ilegal de la madera en la Comunidad de Río Blanco, Provincia de Napo, año 2016. Universidad Central del Ecuador. p. 1-91. http://www.dspace.uce.edu.ec/handle/25000/15485

Uriarte, J. (2021). Definición y características Deforestación. caracteristicas.co. https://www.caracteristicas.co/deforestacion/\#site-header

Uyar, M. (2020). The Association Between Environmental Strategies and Sustainability Performance in the Context of Environmental Management Accounting. Ege Academic Review, 20(1), p. 21-41. https://doi.org/10.21121/eab.590348 
Valderrama-López, C., Ortiz-Avilés, J., Usa-Peña, M. y Fierro, O. (2020). Design of an educational strategy for environmental school programs in Colombia. Scientia et Technica, 25(4), p. 576-583. https://doi.org/10.22517/23447214.22261

Valdez, M., y Cisneros, P. (2020). Gobernanza ambiental, Buen Vivir y la evolución de la deforestación en Ecuador en las provincias de Tungurahua y Pastaza. Revista de Derecho, 34. p. 147-168. https://doi.org/10.32719/26312484.2020.34.8

Velásquez, L. (2019). Create Environmental Leaders From The Ethics, Implementing Innovative Pedagogical Strategies. Journal of Alternative Perspectives in the Social Sciences, 10(1).p. 73 - 81. https://bit.ly/3z1swXO

Vivanco, G. (2019). Modelamiento Espacial en la Distribución Potencial de Cedrelinga Cateniformis Ducke (Tornillo) y Cunuria Spruceana Baillon (Higuerilla) En los Bosques de Producción Permanente, Distrito la Morada, Huanuco. Universidad Nacional Agraria De La Selva. p. 1-108. http://repositorio.unas.edu.pe/handle/UNAS/1604?show=full

Voda, M., Torpan, A. y Moldovan, L. (2017). Wild Carpathia Future Development: From Illegal Deforestation to ORV Sustainable Recreation. Sustainability, 9(12), p. 2254. http://dx.doi.org/10.3390/su9122254

Win, Z., Mizoue, N., Ota, T., Wang, G., Innes, J., Kajisa, T. y Yoshida, S. (2018). Spatial and Temporal Patterns of Illegal Logging in Selectively Logged Production Forest: A Case Study in Yedashe, Myanmar. Journal of Forest Planning, 23(2), p. 15-25. https://bit.ly/2SRPYWS

Zhang, X., Xu, B., Wang, L., Yang, A. y Yang. H. (2016). Eliminating Illegal Timber Consumption or Production: Which Is the More Economical Means to Reduce Illegal Logging? Forests (19994907), 7(9), p. 191. https://doi.org/10.3390/f7090191 